\title{
Contribution à la connaissance du genre Baetis Leach, 1815 en Corse (Ephemeroptera ; Baetidae). B. albinatii nov. sp. du groupe muticus (L.)
}

\author{
M. Sartori 1 \\ A.G.B. Thomas 2
}

Mots clés : Ephemeroptera, Baetidae, espèce nouvelle, Corse.

Une espèce nouvelle, Baetis albinatii nov. sp., est décrite (imago mâle et larve au dernier stade) à partir de matériel récolté en Corse. Elle appartient au groupe muticus et est très voisine de B. navasi Müller-Liebenau, 1974.

Contribution to the knowledge of the genus Buctis Leach, 1815 in Corsica (Fphemeroplera ; Baetidae). B. albinatii nov. sp. of the muticus group.

Keywords : Ephemeroptera, Baetidae, new species, Corsica.

A new species, Baetis albinatii nov. sp., is described (male imago and last instar larva) from material collected in Corsica. This species belongs to the muticus group and is closely related to B. navasi Müller-Liebenau, 1974.

\section{Introduction}

La faune des Ephéméroptères de Corse a fait l'objet ces dernières années de plusieurs travaux (Thomas \& Gazagnes 1984, Sartori 1986, Sartori \& Jacob 1986, Belfiore 1987, Thomas \& Soldán 1987). Ces études ont permis la redécouverte d'espèces anciennes, ou la description de taxa nouveaux.

La Corse présente en réalité un taux d'endémisme beaucoup plus important qu'il n'a généralement été admis auparavant. Dans le genre Baetis notamment, seules deux especes sont actuellement décrites avec assez de précision pour pouvoir ètre identifiées sans ambiguité. Il s'agit de $B$. cymetus Thomas \& Gazagnes, 1984 et de $B$. ingridae Thomas \& Soldán, 1987.

1. Musée Zoologique. Place Riponne 6, CP $448, \mathrm{CH}-1000$ Lausanne 17. Suisse.

2. Laboratoire d'Hydrobiologie. UA 695 du CNRS. Université Paul Sabatier, 118, route de Narbonne, F-31062 Toulouse Cédex, France.
La première est vicariante de B. alpinus (Pictet), la deuxième de B. rhodani (Pictet).

D'autres espèces du gen re Baetis ont été signalées de Corse, mais sans que leur appartenance spécifique puisse être formellement démont rée. Ce sont : B. mutictus (L.) $(=$ B. pumilus Burm.), B. fuscaus (L.) $(=B$. binoculatus L.), $B$. niger $($ L.) et $B$. vermus Curt. (voir: Hagen 1864, Esben-Petersen 1912, 1913, Klapalek 1917, Kimmins 1930, Verrier 1954, Orsini 1986).

Des prospections effectućes par l'un de nous (M.S.) dans certains cours d'eau corses pendant l'été 1985 et le printemps 1988 ont permis la récolte d'un nombre important de larves et d'adultes proches de $\boldsymbol{B}$. muticus.

Une étude détaillée a montré qu'il ne s'agissait pas de l'espèce décrite par Linné, mais d'un taxon nouveau pour la science dont les caractères morphologiques l'apparentent d'ailleurs davantage à $B$. navasi Müller-Liebenau, 1974. 


\section{Baetis albinatii nov, sp. : description}

Cloe pumila Burm. sensu Hagen, 1864.

Baetis pumilus Burm. sensu Esben-Petersen, 1913.

Baetis pumilus Burm. sensu Klapalek, 1917.

Baetis muticus L. sensu Orsini, 1986.

\section{Larve au demier slade}

Diagnose sommaire: espèce typiquement du groupe muticus, à thorax nettement comprimé latéralement, et à languette postéro-interne sur chaque paraprocte ; 6 paires de branchies abdominales.

\section{Taille}

Longueur du corps: $5,9-6,8 \mathrm{~mm}\left(\sigma^{\circ}\right)$; $6,1-7,9 \mathrm{~mm}(9)$

Longueur des cerques: $3,6-5,1 \mathrm{~mm}\left(\sigma^{\prime}\right)$; $3,8-5,3 \mathrm{~mm}(\wp)$

Longueur du paracerque: $2,5-3,1 \mathrm{~mm}\left(\sigma^{\circ}\right)$; 2,6-3,6 mm (q).

\section{Tềte}

Coloration allant du brun moyen chez les femelles, au brun foncé chez les mâles. Traces d'insertions musculaires très peu contrastées. Antennes : pédicelle (fig. 1) portant beaucoup moins de traces incurvées que chez $B$. navasit, et dont la surface apparaît légèrement réticulée par endroits.

Labre : rapport $\mathrm{l} / \mathrm{L}$ compris ent re 1,3 et 1,4 , soit sensiblement le même que pour $B$. muticus et $B$. navasi. Nombre de grandes soies frontales: $1+(1-2)$. Surface du labre couverte de fines soies, surtout dans la moit ié distale. Près de la marge latérale, 3-6 épines disposées régulièrement. Soies marginales antéro-latérales plus ramifiées que celles de $B$. navasi (fig. 2).

Mandibules de morphologie voisine de celles des autres espèces du groupe. Plusieurs denticules bien marqués et aigus près des soies correspondant à la prostheca droite.

Maxilles: palpe régulièrement arrondi à l'apex, sans aucune écaille ou dent apicale.

Labium : $3^{\mathrm{e}}$ article régulièrement arrondi du côté externe et à contour interne légèrement convexe ou subrectiligne près de l'apex (fig. 3), ce qui peut le distinguer quelque peu de celui des espèces précédentes, plutôt légèrement concave (voir Müller-Ljebenau 1969, fig. 143c et Müller-Liebenau 1974, fig. 14c). Paraglosses et glosses assez semblables à celles de $B$. muticus et de B. navasi.
Thorax

Pronotum brun moyen à brun foncé avec des zones claires médiane et latérales assez comparables à celles de $B$. muticus, mais en général la tache médiane est plus large vers l'arrière (fig. 4). Pattes gris jaunâtre. Une large bande brune préapicale sur les fémurs des exemplaires les plus pigmentés.

Bord postéro-externe des fémurs avec de fortes soies coniques semblables à celles de $B$. muticus. Ces soies sont nettement moins abondantes chez B. albinatii que chez $B$. navasi, tant sur la marge du fémur (fig. 5) et en particulier vers l'apex, que sur celle du tibia. Les différences avec $B$. navasi sont surtout perceptibles sur les pattes antérieures et médianes (Tableau I). D'autre part, la surface supérieure du fémur, qui apparaît légèrement réticulée chez $B$. albinatii, porte peu de traces incurvées (davantage sur la face inférieure pour $\mathrm{P} 1$ ) comparativement à B. navasi.

Griffe tarsale armée de 10 à 13 dents (fig. 6).

\section{Abdomen}

Coloration dorsale brun foncé assez uniforme. Deux taches latérales blanches sur le tergite I. Dans la partie médiane des tergites II - rarement I - à VI inclus, une tache claire assez semblable à celle rencontrée chez $B$. muticus. L'étendue de cette tache peut varier sensiblement d'intensité d'un individu à l'autre, jusqu'à devenir imperceptible (fig. 7). Chez les spécimens peu contrastés, les traces d'insertions musculaires sont alors bien visibles et semblables à celles de $B$. navasi. Tergites IX et $\mathrm{X}$ en règle générale beaucoup plus clairs que les précédents et plus ou moins largement blancs.

6 paires de branchies, comme chez B. navasi : la première fait défaut (fig. 8). Sur le premier segment des larves âgées, l'emplacement correspondant à l'articulation branchiale est légèrement saillant et noirci ponctuellement. Forme des branchies assez semblable à celle des deux autres espèces du groupe muticus. Bord branchial nettement ourlé de brun soutenu, et complètement dépourvu de traces incurvées sur les 2/3 distaux (fig. 9). Chez $B$. navasi, ces traces sont plus serrées et présentes jusqu'à l'apex.

Bord postérieur des tergites avec des dents triangulaires, aiguës, plus étroites que celles de $B$. navasi (fig. 10). L'ornementation cuticulaire des tergites, en particulier les traces d'insertions d'écailles, est 
0.1

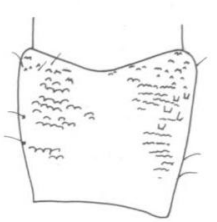

1 a

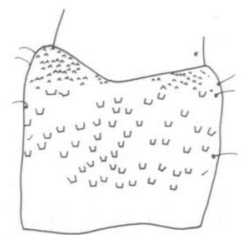

$\mathrm{n}$

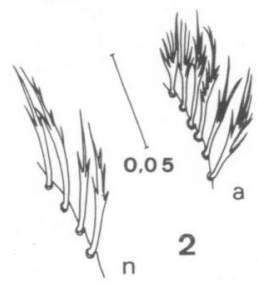

5 a

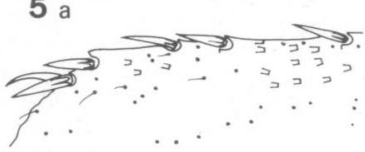

0,1

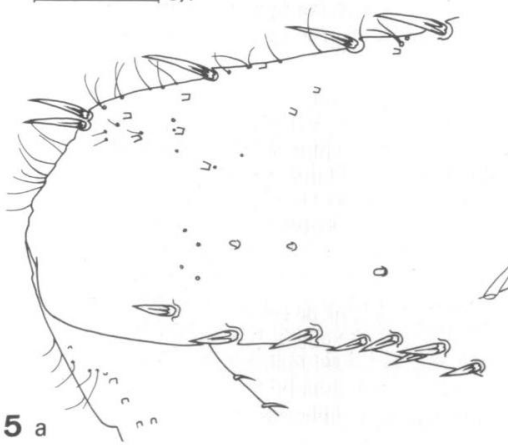

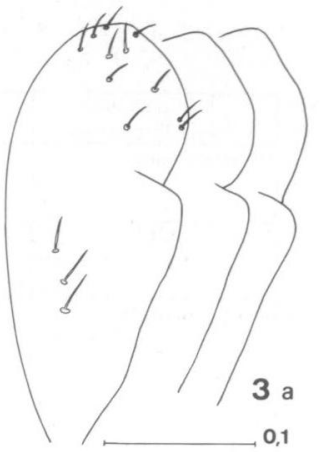

0,5

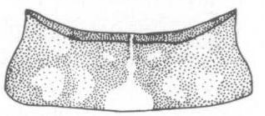

4 a
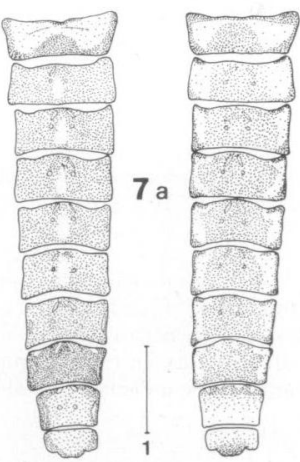

Fig. 1 a 7 : larves de Baetis albinatil n.sp. (a) et de B. ravasi (n) au dernier stade. Echelle en mm. 1: pédicelle. -2 : soies marginales antéro-latérales du labre. $-3: 2^{c}$ et $3^{c}$ articles du palpe labial sur 3 exemplaires. - 4: coloration du pronotum. -5 : région distale du fémur $1 .-6$ : griffe tarsale. -7 : coloration dorsogabdominale. 
Tableau I.

\begin{tabular}{|c|c|c|c|c|c|}
\hline \multirow{2}{*}{$\begin{array}{c}\text { Soies coniques } \\
\text { marginales externes }\end{array}$} & \multicolumn{2}{|c|}{ B. albinatii } & \multicolumn{2}{|c|}{ B. navasi } & \multirow{2}{*}{$\begin{array}{c}\text { Test de Student } \\
\text { p }\end{array}$} \\
\hline & total & moyenne & total & moyenne & \\
\hline $\begin{array}{ll}\text { Pattes antérieurcs } & \\
& \text { Fém }\end{array}$ & $\begin{array}{c}13-23 * \\
4-11\end{array}$ & $\begin{array}{c}15 \\
7 \\
\end{array}$ & $\begin{array}{l}23-32 \\
19-23 \\
\end{array}$ & $\begin{array}{l}26 \\
21 \\
\end{array}$ & $\begin{array}{l}<0.001 \\
<0.001 \\
\end{array}$ \\
\hline Pattes médianes & $\begin{array}{l}10-17 \\
9-21 * *\end{array}$ & $\begin{array}{l}14 \\
13\end{array}$ & $\begin{array}{r}16-45 \\
22-32 \\
\end{array}$ & $\begin{array}{l}26 \\
27 \\
\end{array}$ & $\begin{array}{l}<0.05 \\
<0.001 \\
\end{array}$ \\
\hline \begin{tabular}{rr|} 
Pattes postérieures & \\
& Fémurs \\
& Tibias \\
\end{tabular} & $\begin{array}{r}10-21 \\
7-13 \\
\end{array}$ & $\begin{array}{l}13 \\
11 \\
\end{array}$ & $\begin{array}{l}13-24 \\
15-20 \\
\end{array}$ & $\begin{array}{l}19 \\
19 \\
\end{array}$ & $\begin{array}{r}<0.05 \\
<0.001 \\
\end{array}$ \\
\hline
\end{tabular}

* 13.14 soies sur 7 exemplaires, 23 sur un seul

* 9 -16 soies sur 7 exemplaires, 21 sur un seul

proche de celle de cette demière, mais la surface des tergites est nettement plus striée transversalement.

Paraproctes comparables aussi à celles de $B$. navasi, en particulier par la présence de fortes épines sur la surface du prolongement postéro-interne (fig. 11). Les épines marginales de ce prolongement sont au moins aussi longues que chez $B$. navasi et donc nettement plus que chez $B$. muticus -, mais plus nombreuses et plus serrées parce que plus étroites (fig. 12).

Cerques clairs, brun jaunâtre, plus intensément colorés en brun moyen dans la partie proximale.

Imago or

Taille

Corps : $6,2-6,6 \mathrm{~mm}$; aile antérieure : 5,2-5,5 mm ; cerques : $9,7-10,1 \mathrm{~mm}$.

Tête

Face brun soutenu. Antennes : scape et pédicelle brun moyen ; flagelle brun gris renflé à la base. Ocelles gris blanchâtre cerclés de brun noir. Surface dorsale des yeux en turban brun orangé. Pédoncule orange avec un anneau basal plus foncé.

\section{Thorax}

Coloration générale brun foncé. Membranes pleu. rales brun clair. Fémurs et tibias de toutes les pattes de couleur blanchâtre. Tarses grisâtres ; articulation fémoro-tibiale légèrement teintée de brun clair. Aile antérieure entièrement hyaline à nervation alaire peu contrasté, avec tout au plus les nervures longitudinales légèrement teintées de beige dans leur partie proximale. Aire ptérostigmatique laiteuse avec 5 à 10 nervures transverses simples et peu visibles. Aile postérieure hyaline avec 3 nervures longitudinales, la $2^{e}$ bifurquée. Processus costal bien marqué. 0 à 2 nervures transverses difficilement visibles (fig. 13).

\section{Abdomen}

$1^{\text {er }}$ segment abdominal brun foncé. Segments IIVI translucides; VII-X colorés en brun moyen, les sternites un peu plus clairs que les tergites. $I{ }^{c}$ sternite assez semblable à celui de $B$, navasi, mais les plaques internes en sont net tement moins chitinisées (fig. 14). Cerques blanchátres sur toute leur longueur.

\section{Genitalia}

Ils sont voisins de ceux de B. muticus. Gonopodes translucides. Segment basal gris beige à brun clair pussédant un léger bourrelet interne arrondi (fig. 14). [er article des gonopodes nettement conique. Dernier article subsphérique, légèrement plus long que large.

Imago $q$, subimagos $\%$ et $\%$ inconnues.

\section{Affinités}

Par ses stades larvaire et imaginal, $B$. albinatii nov. sp. appartient au groupe muticus. 

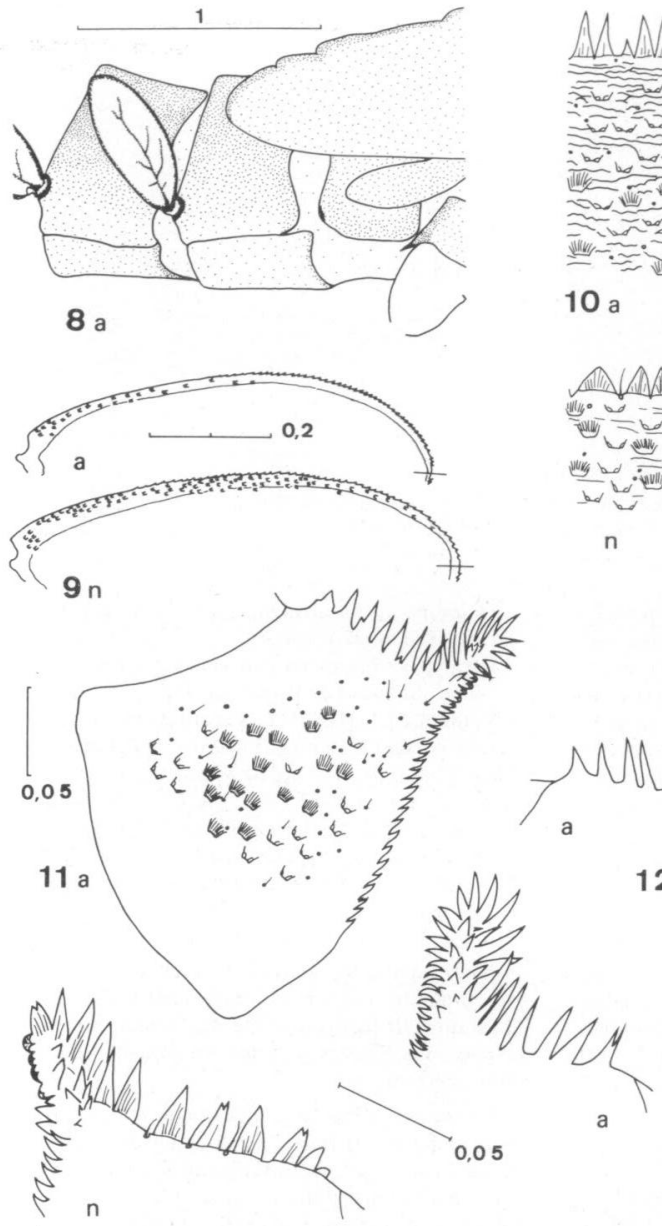

Fig. 8 à 12 : larves de Baetis albinatii n. sp. (a) et de B. navasi (n) au dernier stade. Echelle en mm.

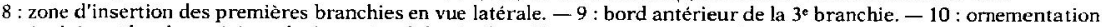
cuticulaire et bord postérieur du 4 * tergite abdominal. - 11 : paraprocte. - 12 : languette postéro-interne et bord postérieur de la paraprocte sur plusieurs exemplaires.

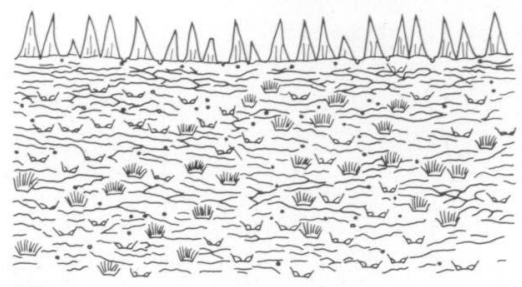

10 a

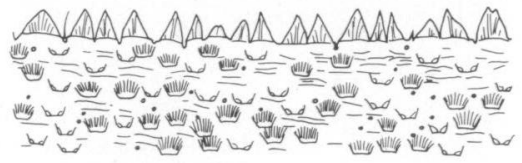

$\mathrm{n}$ 0,05 0,05

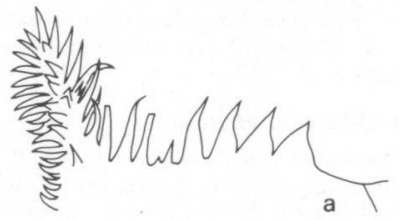



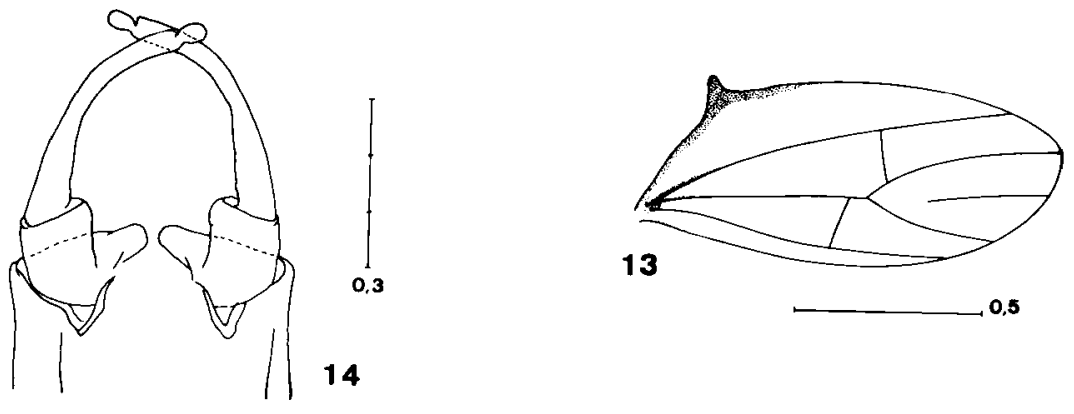

Fig. 13 et 14 : imagos $\sigma$ de Baetis albinatii $n$. sp. Echelle en mm. 13: aile postérieure. -14 : genitalia en vue ventrale.

Au stade larvaire, et malgré la coloration abdominale très semblable, $B$. albinatii se distingue aisément de $B$. muticus par l'absence de la première paire de branchies. Elle diffère de $B$. navasi surtout par la forme des épines marginales des tergites abdominaux et des paraproctes. par l'ornementation des pattes antérieures et médianes, et quelque peu aussi par son labium et par la coloration de ses tergites abdominaux.

Au stade imaginal, $B$. albinatii est proche de $B$. muticus et aussi de $B$. navasi dont l'imago a été décrite par Müller-Liebenau (1976). Elle paraît présenter quelques différences minimes vis-à-vis de ces deux especes, respectivement sur la forme et la chitinisation des plaques du IX ${ }^{c}$ sternite abdominal $(B$. muticus), et sur la forme de l'article basal des gonopodes (B. navasi) : voir Müller-Liebenau (op. cit.), p. 67 et 68 .

\section{Matériel examiné}

a) Baetis navasi Müller-Liebenau

Portugal, Serra da Estrela (coll. J. Aubert) :

4 larves (L), 2 larves âgées (N), de Gouveia au col de San Lorenzo, 1300 m, 7.VI.1959; 1 N, 1 L, torrent de Valezim, $800 \mathrm{~m}, 19 . \mathrm{IV} .1960 ; 1 \mathrm{~N}, 1 \mathrm{~L}$, Cabeça da Velho, ruisselet à $1300 \mathrm{~m}, 17 . I V .1960$.

b) Baetis albinatii nov. sp.

Corse (coll. M. Sartori) :
Holotype : $1 \mathrm{~N}$, affluent du Taravo, amont de Zicavo, $800 \mathrm{~m}, 22 . \mathrm{V} .1988$.

Paratypes : $5 \mathrm{~N}$, mêrme provenance ; $4 \mathrm{~N}$, Gravona, versant sud du col de Vizzavona, $900 \mathrm{~m}$, 5.VII.1985, $1 \mathrm{~N}$, idem, 21.V.1988; $2 \mathrm{~L}$, Vecchio, aval de la voie ferrée (col de Vizzavona), $1000 \mathrm{~m}, 5$. VII. 1985, $22 \mathrm{~N}$, idem, 21.V.1988; $1 \mathrm{~N}$, col de Vizzavona, cascade des Anglais, $1100 \mathrm{~m}, 5$. VII.1985; 1 N, Gravona, aval cascade du Voile de la Mariée, $950 \mathrm{~m}, 5$. VII. $1985 ; 3 \mathrm{~N}$, $5 \sigma^{\circ}$, affluent du Taravo, aval de Zicavo, $500 \mathrm{~m}$. $22 . V .1988 ; 1 \sigma$, affluent du Taravo, confluence avec un 2 affluent, $500 \mathrm{~m}, 22 . \mathrm{V} .1988 ; 3 \mathrm{~N}$, Taravo, route Forciolo-Olivese, $240 \mathrm{~m}, 7$. VII. 1985 .

Nous rapportons aussi à cette espèce quelques larves, aimablement communiquées par le Professeur J. Giudicelli (Université de Marseille), que nous remercions. Elles proviennent de la Restonica entre 400 et $800 \mathrm{~m}$.

L'holotype, ainsi que la majorité des paratypes sont déposés au Musée zoologique de Lausanne. Quatre paratypes se trouvent dans la collection Thomas à Toulouse; deux autres sont déposés au Muséum d'Histoire Naturelle de Genève.

\section{Ecologie sommaire}

B. albinatii nov. sp. a été capturée dans de petits cours d'eau caractéristiques de l'épi- et du méta. rhithron, a des altitudes comprises entre 240 et 
$1100 \mathrm{~m}$. La temperature de l'eau au moment des récoltes variait entre 8 et $11^{\circ} \mathrm{C}$ au mois de mai, et entre 13 et $20^{\circ}$ au mois de juillet. Cette espece n'est pas rare, et dans certains cours d'eau comme le Vecchio par exemple, B. albinatii est mème l'espèce du genre dominante au printemps. Comme espèces accompagnatrices, récoltées au mème moment, on peut citer $B$. cyrneus et $B$. ingridae. Cette association correspondrait donc à celle de B. alpinus, B. rhodani et $B$. muticus, fréquente dans les cours d'eau collinéens et montagnards d'Europe occidentale et centrale.

D'après Orsini (1986), B. albinatii (s.n. B. muticus) est présente également dans l'Orta, le Zingaju et la Rizzanese, à des altitudes sensiblement comparables.

Quant au cycle de développement de cette espèce, nos captures au printemps 1988 et en été 1985 tendraient à mont rer que B. albinatii est probablement une espèce multivoltine. Selon Orsini (op. cit.), les larves de $B$. albinatii sont présentes toute l'année dans la Rizzanese et la Restonica, ce qui indiquerait que cette espèce a probablement deux générations dans l'année, qu'elle ne passe pas l'hiver en diapause embryonnaire, mais sous forme de larve, ce qui en ferait donc une espèce hiverno-estivale (MBws au sens de Clifford, 1982).

\section{Remerciements}

Nos plus sincères remerciements vont à notre ami. le $D^{x} D$. Cherix (Lausanne) pour son aide efficace sur le terrain. Notre reconnaissance va également à $\mathbf{M}$. S. Albinati (Monaco) sans lequel ce travail n'aurait pu étre réalisé. C'est avec plaisir que nous lui dédions cet te nouvelle espèce. Nous remercions également le $D^{r}$ B. Hauser (Museum d'Histoire Naturelle de Genève) pour nous avoir aimablement prêté l'holotype de B. navasi. Le voyage en Corse du premier auteur en 1985 a pu étre réalisé gráce à une bourse de voyage de la Société Helvétique des Sciences Naturelles.

\section{Travaux dites}

Belfiore (C.). 1987. - Heptageniidae from Corsica and Sardinia. Rhithrogena nuragica n. sp., R. eatoni Esben-Petersen 1912, and R. insularis Esben-Petersen 1913 (Ephemeroptera). Annls Lim. nol, 23 (2) : 87-94.

Clifford (H.C.). 1982. - Life cycles of maytlies, with special reference to voltinism. Quest. Ent., 18 (1-4) : 15-90.

Esben-Petersen (P.). 1912. - Addition to the knowledge of the Neu. ropterous insect fauna of Corsica. Ent. Medd., 9: 348-353.

Esben-Petersen (P.). 1913. - Addition to the knowledge of the Neuropterous insect fauna of Corsica II. Ent. Medd., $10: 20-28$.

Hagen (H.A.). 1864, - Neuroptères (non Odonates) de la Corse, recueillis par M.E. de la Chavignerie en 1860 et 1861 . Annls Soc. ent. Fr., $4: 38.39$.

Kimmins (D.E.). 1930, - A list of the corsican Ephemeroptera and Neuroptera. Eus, 6 (2) : 185-190.

Klapalek (F.). 1917. - Über die von Herm Prof. A. Hetschko in Korsika gesammelten Neuropteroiden nebst Bemerkungen über einige ungenügend bekannte Arten. Wiener Ent. Zeit., 36: 193-208.

Möller-Liebenau (I.). 1969. - Revision der europäischen Arten der Gattung Baetis Leach, 1815 (Insecta, Ephemeroptera), Gewäss. Abwäss., $48 / 49: 1.214$.

Müller-Liebenau (I.). 1974, - Baetidae aus Süd-Frankreich, Spanien und Portugal. Gewäss. Abuäss, 53/54: 7-42.

Müller-Liebenau (I+). 1976. - Die Imagines ron Baetis navasi Müller-Liebenau 1974 aus Portugal (Insecta, Ephemeroptera). Gewäss Abwäss., 60/61 : 65-69.

Orsini (A.). 1986. - Influence du couvert végétal du bassin versant sur les caractéristiques thermiques, chimiques et biologiques des cours d'eau de Corse. Thèse d'Erat, Univ. Aix-Marseille III, $230 \mathrm{p}$.

Sartori (M.). 1986. - Révision taxonomique du genre Habroleptoi. des Schönemund, 1929 (Ephemeroptera, Leptophlebiidae). III : Description de $H$. annae n. sp. et $H$. thomasi n. sp. et synthèse finale des stades ailés. Revue suisse Zool., 93 (4) : 919-949.

Sartori (M.) et Jacob (U.). 1986. - Révision taxonomique du genre Habroleptoides Schönemund. 1929 (Ephemeroptera, Leptophlebiidae), Il : A propos du statut de Habroleproides modesta (Hagen, 1864). Revue suisse Zool, 93 (3) : 683-691.

Thomas (A.G.B.) et Gazagnes (G.). 1984. - Batis cymeus n. sp., Ephéméroptère nouveau de Corse (Baetidae). Annls Limnol., 20 (3) : 199-202.

Thomas (A.G.B.) et Soldán (T.). 1987. - Baetis ingridae n. sp., Ephé. méroptère nouveau de Corse (Baetidae). Annls Limnol., 23 (1): 23-26.

Verrier (M.L.). 1954. - Contribution à l'étude de la faune d'eau douce de Corse: Ephéméroptères. Vie Milieu, 5 (2) : 280-290. 